Indian J. Phys. 83 (4) 517-523 (2009)

\title{
Structural and magnetic properties of Fe doped $\mathrm{NiO}$
}

\author{
P Mallick ${ }^{1 *}$, Chandana Rath ${ }^{2}, \mathrm{R} \mathrm{Biswal}^{3}$ and N C Mishra ${ }^{3}$ \\ ${ }^{1}$ Department of Physics, North Orissa University, Baripada-757 003, Orissa, India \\ ${ }^{2}$ School of Material Science \& Technology, Institute of Technology, \\ Banaras Hindu University, Varanasi-221 005, Uttar Pradesh, India \\ ${ }^{3}$ Department of Physics, Utkal University, Bhubaneswar-751 004, Orissa, India \\ Email : pravanjan_phy@yahoo.co.in
}

\begin{abstract}
Mott-Hubbard anti-ferromagnetic insulator, NiO shows $p$-type semiconducting behaviour due to vacancy at $\mathrm{Ni}^{2+}$ site in its bunsenite structure. We report the modification of structural and magnetic order in $\mathrm{NiO}$ on $\mathrm{Fe}$ doping. NiO samples at different $\mathrm{Fe}$ concentrations in the range 0 to 5 at.\% have been prepared by chemical co-precipitation and post thermal decomposition method. Both structural and magnetic characterization reveal that with increasing $\mathrm{Fe}$ doping concentration, $\mathrm{NiO}$ evolves as a magnetically inhomogeneous state out of the parent homogeneous antiferromagnetic state. In addition, structural inhomogeneity was also observed with Fe precipitating to $\gamma-\mathrm{Fe}_{2} \mathrm{O}_{3}$ phase, the signature of which could be clearly seen for $\mathrm{Fe}$ content beyond 2 at.\%. At lower Fe content however, some amount of $\mathrm{Fe}$ occupies lattice and interstitial sites in the $\mathrm{NiO}$ matrix and drive the latter to acquire ferromagnetic ordering, which was evident from a clear hysteresis loop at $300 \mathrm{~K}$.
\end{abstract}

Keywords : Dilute magnetic semiconductor, X-ray diffraction, magnetization, NiO.

PACS Nos. : 91.60.Ed, 96.12.Hg, 61.10.Nz, 61.72.Vv, 91.25.Rt

\section{Introduction}

Recent years have seen an upsurge of research in Diluted Magnetic Semiconductor (DMS) materials due to their application in spintronic devices [1]. DMS has charge and spin degrees of freedom in a single substance, leading to novel applications combining magnetic, electronic and optical functionalities. The discovery of room temperature ferromagnetism in $\mathrm{Co}^{2+}$ doped $\mathrm{TiO}_{2}, \mathrm{Mn}^{2+}$ and $\mathrm{Ni}^{2+}$ doped $\mathrm{ZnO}$ are due to charge carrier electron, known as $n$-type DMS materials. As compared to the $n$-type DMS systems like transition metal (TM) doped $\mathrm{ZnO}$ and $\mathrm{TiO}_{2}$, there exist a few $p$-type DMS systems, the detailed characteristics of which have not yet been reported. A theoretical prediction by Dietl et al, [2] indicates that the curie temperature of $p$-type DMS can be well above that of $n$-type DMS. 
$\mathrm{NiO}$ is a $p$-type semiconductor due to vacancy at $\mathrm{Ni}^{2+}$ sites [3,4]. It is an antiferromagnetic Mott-Hubbard insulator with Neel temperature, $T_{N}=523 \mathrm{~K}$. The magnetic structure of $\mathrm{NiO}$ consists of ferromagnetic sheets of $\mathrm{Ni}^{2+}$ parallel to the (111) plane with opposite spin directions in neighbouring sheets. TM doping [5] or reduction of particle size [6] have been shown to disturb the magnetic structure of $\mathrm{NiO}$ with evolution of ferromagnetism in some cases.

The hole content in the p-type $\mathrm{NiO}$ is enhanced on TM doping, which is believed to cause the observed DMS in this system. Nowotny et al [7] reported p-type properties in $\mathrm{Cr}$ doped $\mathrm{NiO}$. Battle et al [8] reported that Co doping doesn't induce ferromagnetic ordering in $\mathrm{NiO}$. Instead the $T_{N}$ increases linearly with Co concentration. $\mathrm{Fe}$ doped $\mathrm{NiO}$ is a $p$-type material, which has been shown to exhibit ferromagnetism at room temperature [5]. The basic crystal structure of $\mathrm{NiO}$ (bunsenite) being different from that of $\mathrm{FeO}$ (wustite), $\mathrm{Fe}$ doping in $\mathrm{NiO}$ matrix is expected to create considerable strain in the latter. The associated modification in $\mathrm{Fe}$ doped $\mathrm{NiO}$ should therefore influence its magnetic properties. In the present study, we have examined both the magnetic and the structural properties of $\mathrm{Fe}(0$ to 5 at.\%) doped $\mathrm{NiO}$. At high $\mathrm{Fe}$ concentration, though second phase precipitation leads to formation of $\gamma-\mathrm{Fe}_{2} \mathrm{O}_{3}$, the observed magnetization at low Fe concentration ( $<3$ at.\%) is found to be a consequence of $\mathrm{Fe}$ occupying the $\mathrm{NiO}$ matrix both at lattice sites as well as at interstitial positions.

\section{Experimental}

The $\mathrm{Ni}_{1-x} \mathrm{Fe}_{x} \mathrm{O}$ samples with $x$ varying from 0 to 5 at.\% were prepared by chemical coprecipitation method. Stoichiometric amount of $\mathrm{Ni}\left(\mathrm{NO}_{3}\right)_{2} \cdot 6 \mathrm{H}_{2} \mathrm{O}$ and $\mathrm{Fe}\left(\mathrm{NO}_{3}\right)_{3} \cdot 9 \mathrm{H}_{2} \mathrm{O}$ were dissolved in distilled water. The hydroxycarbonate precursor was chemically precipitated by slowly adding an aqueous $\mathrm{NH}_{4} \mathrm{HCO}_{3}$ solution of $\mathrm{pH} \sim 8$ at room temperature accompanying magnetic stirring. The resultant gels were washed several times with distilled water and ethanol until free of nitrate ions, filtrated and dried in air at $353 \mathrm{~K}$. The dried gels were then calcined at $873 \mathrm{~K}$ for 4 hours in air to obtain Fedoped $\mathrm{NiO}$ samples. XRD measurements were done at $300 \mathrm{~K}$ using Rigaku Powder Diffractometer with $\mathrm{CuK}_{\alpha}$ radiation. Magnetic properties of different $\mathrm{Fe}$ doped $\mathrm{NiO}$ samples were studied using Physical Properties Measurement System (PPMS)-VSM.

\section{Results and discussion}

\subsection{Structure and microstructure evolution :}

Since the radius of $\mathrm{Fe}^{3+}$ ion $(0.64 \AA)$ is almost same as that of $\mathrm{Ni}^{2+}$ ion $(0.69 \AA), \mathrm{Fe}$ is expected to substitute $\mathrm{Ni}$ in the lattice structure of $\mathrm{NiO}$. XRD plots (Figure 1) show that $\mathrm{Ni}_{1-x} \mathrm{Fe}_{x} \mathrm{O}$ samples remain single phase for $x$ up to 0.02 . Due to limited solubility however, second phase precipitation is expected at higher concentrations of $\mathrm{Fe}$ in $\mathrm{NiO}$ [9]. Appearance of $\mathrm{Fe}_{2} \mathrm{O}_{3}$ phase beyond 2 at.\% and $\mathrm{NiFe}_{2} \mathrm{O}_{4}$-phase beyond 5 at.\% of 


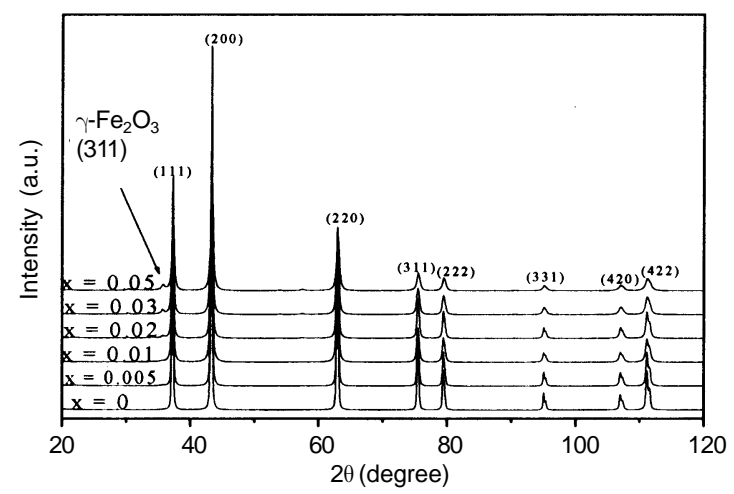

Figure 1. XRD pattern of $\mathrm{Ni}_{1-x} \mathrm{Fe}_{x} \mathrm{O}(x=0$ to 0.05$)$.

$\mathrm{Fe}$ in $\mathrm{NiO}$ has been reported [5]. Our XRD plots for $x>0.02$ (Figure 1) show that the (311) peak of $\gamma-\mathrm{Fe}_{2} \mathrm{O}_{3}$ appear as the second phase. The FCC structure of host $\mathrm{NiO}$ phase however does not change on $\mathrm{Fe}$ doping. The lattice parameter remains almost same $(4.176 \AA)$ up to 3 at.\% $\mathrm{Fe}$ doped $\mathrm{NiO}$ samples whereas it shows a slight increase for 5 at.\% Fe concentration.

Figure 2 shows the increase of line width at different peak positions corresponding to their (hkl) with increasing $\mathrm{Fe}$ content in NiO. Wang et al [5] from a similar observation of XRD line broadening, calculated particle size using Debye-Sherrer formula and concluded that size of $\mathrm{NiO}$ particles reduces from 60 to $35 \mathrm{~nm}$ on increasing $\mathrm{Fe}$ content from 0 to 2 at.\%. The basic crystal structure of $\mathrm{NiO}$ (bunsenite), however is

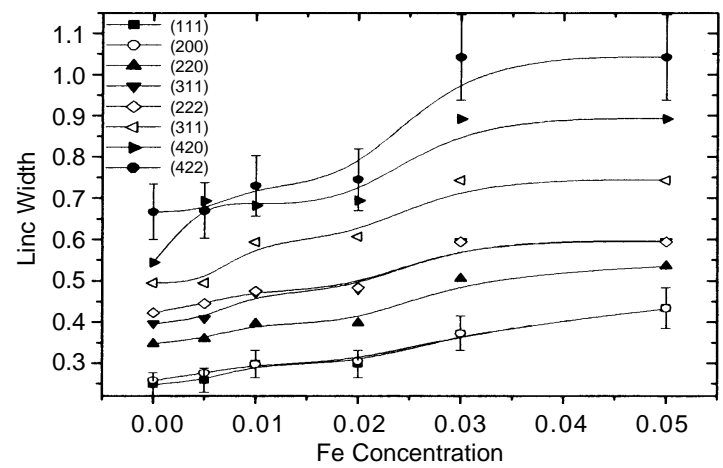

Figure 2. The variation of line width with various Fe concentrations at different peak position indicated by their $(h k l)$.

different from that of $\mathrm{FeO}$ (wustite). Fe doping therefore is expected to create a large amount of strain in $\mathrm{NiO}$ lattice. Hence use of Debye-Sherrer formula without taking strain into consideration for calculation of particle size may lead to erroneous result. Apart from the contribution of particle size reduction, defects and disorder in the lattice affect the width of the XRD lines. The increase in XRD line width with increasing $\mathrm{Fe}$ content in $\mathrm{NiO}$, for example, can arise due to the following possibilities. 
At low concentration, when $\mathrm{Fe}^{3+}$ occupies $\mathrm{Ni}$ site, two such $\mathrm{Fe}$ ions can lead to a vacancy at $\mathrm{Ni}$ site [5]. $\mathrm{Cr}$ doped $\mathrm{NiO}$ also presents a similar scenario [7]. The associated strain at the substituted $\mathrm{Fe}$ sites as well as at the vacant $\mathrm{Ni}$ site can contribute to line broadening. Another possibility pertains to the widely different basic structure of $\mathrm{NiO}$ and $\mathrm{FeO}$. Due to two order of larger nonstoichiometry, $\delta\left(\delta \sim 5 \times 10^{-2}\right)$ in the wustite, $\mathrm{Fe}_{1-\delta} \mathrm{O}$ than that in the bunsenite, $\mathrm{Ni}_{1-\delta} \mathrm{O}\left(\delta \sim 5 \times 10^{-4}\right)$, the dopant iron governs the defect structure in $\mathrm{NiO}$, while the later acts like an inert matrix. In this dilute wustite phase of $\mathrm{NiO}$, formation of defect clusters consisting of cation vacancies and iron interstitial is well known $[10,11]$. The basic unit of these clusters consists of four $\mathrm{Ni}$ vacancies tetrahedrally coordinating a $\mathrm{Fe}^{3+}$ interstitial ion. Such clusters were observed by extended X-ray absorption fine structure analysis [12]. These clusters when isolated from each other would lead to extensive strain generation and line width broadening due to four $\mathrm{Ni}$ vacancies for every $\mathrm{Fe}$ ion doped at the interstitial site.

Apart from the contribution of strain induced by $\mathrm{Fe}$ doping in $\mathrm{NiO}$ structure, particle size reduction can lead to broadening of XRD lines as observed by earlier authors [5]. From the broadening of the individual peaks, we calculated the particle size and microstrain values from the Williamson-Hall plot [13]. Particle size shows an almost monotonic decrease with increasing $\mathrm{Fe}$ content from $55 \mathrm{~nm}$ in pure $\mathrm{NiO}$ to 30 $\mathrm{nm}$ in 5 at.\% Fe doped NiO. The mechanism for the suppression of crystal growth of $\mathrm{NiO}$ on Fe doping, which was also observed by Wang et al [5] is not clear. The strain on the other hand increases with increasing Fe content and attains a maximum value for 3 at.\% $\mathrm{Fe}$ in $\mathrm{NiO}$. The rapid increase of strain with increasing $\mathrm{Fe}$ content indicates that most of the $\mathrm{Fe}$ goes to the interstitial site within this concentration range and produces $4 \mathrm{Ni}$ vacancies for every interstitially doped $\mathrm{Fe}$ as discussed earlier. Beyond 3 at.\% $\mathrm{Fe}$, the strain decreases indicating strain relaxation due to phase segregation and formation of $\gamma-\mathrm{Fe}_{2} \mathrm{O}_{3}$ phase as clearly seen in XRD plot for 5 at.\% Fe doped $\mathrm{NiO}$ (Figure 1).

\subsection{Magnetization :}

XRD result indicates that beyond 2 at.\% of $\mathrm{Fe}$ in $\mathrm{NiO}$, phase segregation leads to formation of $\gamma-\mathrm{Fe}_{2} \mathrm{O}_{3}$. We therefore confine the discussion on magnetic characteristics of $\mathrm{Fe}$ doped $\mathrm{NiO}$ for $\mathrm{Fe}$ content up to 2 at.\%. Figure 3 shows the temperature dependence of zero field cooled (ZFC) and field cooled (FC) magnetization in a $500 \mathrm{Oe}$ dc field for 2 at.\% Fe doped $\mathrm{NiO}$. The inset of Figure 3 shows the difference between FC and ZFC magnetization $(\Delta \mathrm{M})$ as a function temperature. The nonzero value of $\Delta \mathrm{M}$ eliminates the para and diamagnetic contributions to the observed magnetic behaviour leaving only the contribution of hysteretic ferromagnetic regime. Generally, the ZFC susceptibility shows a peak for both superparamagnets and spin glasses. It is usually seen that the temperature dependence of the FC susceptibility becomes saturated below the peak temperature (freezing temperature $T_{f}$ ) for spin glasses and continues to 


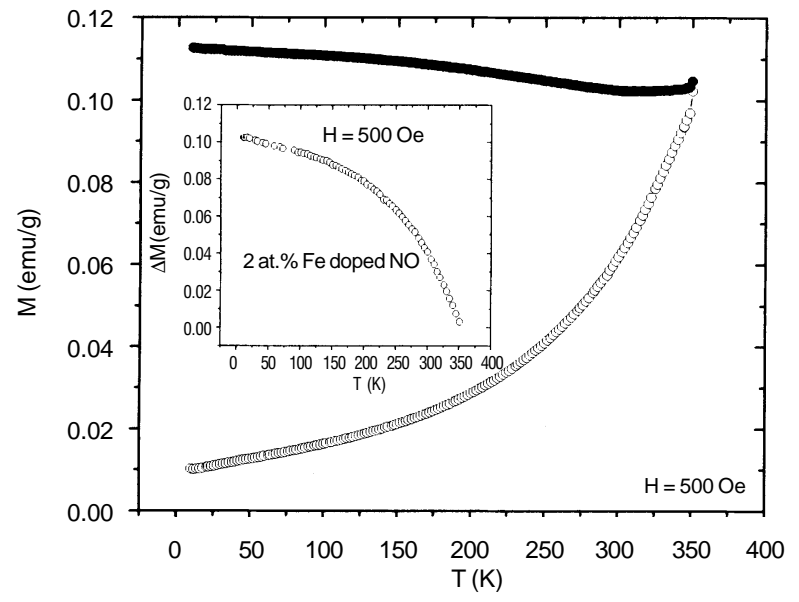

Figure 3. ZFC (open symbol) and FC (solid symbol) magnetization for 2 at.\% Fe doped $\mathrm{NiO}$ particles as a function of temperature in 500 Oe field. The inset shows the difference between FC and ZFC magnetization as a function of temperature.

increase below that temperature (blocking temperature $T_{B}$ ) for superparamagnets [14]. Nevertheless, glassy behavior in magnetic nanoparticles has also been claimed in which the FC susceptibility continues to increase with decreasing temperature [15]. The peak in ZFC magnetization expected for either spin glass or superparamagnetic phases if present in our system does not appear in the measured temperature range from 10 to $350 \mathrm{~K}$. The FC susceptibility shows a slight decrease with decreasing temperature from $350 \mathrm{~K}$ down to $315 \mathrm{~K}$ and then increases with further decreasing temperature up to $10 \mathrm{~K}$. The anomaly around $315 \mathrm{~K}$ in the $\mathrm{FC}$ data suggests $[16,17]$ that some amount of magnetic inhomogeneity might be present in this DMS system.

We now examine any possible contribution that the finite size effect may have to the observed magnetic characteristics in undoped and $\mathrm{Fe}$ doped $\mathrm{NiO}$. Average particle size as depicted from Williamson-Hall plot, ranges from $55 \mathrm{~nm}$ in pure $\mathrm{NiO}$ to $44 \mathrm{~nm}$ in 3 at.\% Fe doped NiO. Kodama et al [6] found anomalous magnetic properties of $31.5 \mathrm{~nm} \mathrm{NiO}$ particles due to finite size effect. These features are absent in large size $\mathrm{NiO}$ particles at room temperature [18]. The $31.5 \mathrm{~nm} \mathrm{NiO}$ particles show a $T_{B} \approx 300 \mathrm{~K}$. In our case, $55 \mathrm{~nm}$ dia NiO particles would thus have a $T_{B}$ much above $300 \mathrm{~K}$. An approximate value of $T_{B}$ can be calculated from the relation [19]

$$
T_{B}=\frac{K V}{25 k_{B}}
$$

where $K$ is the anisotropy constant, $k_{B}$ is the Boltzmann's constant, and $K$ is the volume of the nanoparticle. The above relation gives

$$
\frac{T_{B 1}}{T_{B 2}}=\frac{V 1}{V 2}
$$


where $T_{B 1}, T_{B 2}$ and $V 1, V 2$ are the blocking temperature and volume of particles 1 and 2 respectively. Comparing the volume of the $\mathrm{NiO}$ nanoparticles with a dia of 31.5 $\mathrm{nm}$, which exhibits $T_{B}$ around $300 \mathrm{~K}$ [6] with the volume of our NiO particles, we find $T_{B} \sim 1597 \mathrm{~K}$ for the latter. This huge $T_{B}$, which is much above the $T_{N}$ of $\mathrm{NiO}$, implies that our $\mathrm{NiO}$ particles would exhibit 2-sublattice antiferromagnetic ordering as in bulk $\mathrm{NiO}$. This is in contrast with the finite size effect in nanoparticles of diameter $31.5 \mathrm{~nm}$ and less, where 8-, 6- or 4-sublattice spin configurations arise due to the reduced coordination of surface spins leading to anomalous magnetic properties [6]. Though the particle size of $\mathrm{NiO}$, decreases with increasing $\mathrm{Fe}$ content, it is still much above the value required to see finite size effect for particles up to 3 at.\% Fe doping. At 3 at.\% $\mathrm{Fe}$, the particle size of $44 \mathrm{~nm}$ corresponds to a $T_{B}$ of $818 \mathrm{~K}$, which far exceeds the $T_{N}$ of $\mathrm{NiO}$. The magnetic characteristics that we see in these particles and particles with lower Fe content having still higher particle size, therefore represent bulk behaviour of $\mathrm{Fe}$ doped $\mathrm{NiO}$ and not due to finite size effect.

$\mathrm{M}-\mathrm{H}$ curve obtained from the dc magnetization measurement at $300 \mathrm{~K}$ for $\mathrm{Ni}_{1-\mathrm{x}} \mathrm{Fe}_{\mathrm{x}} \mathrm{O}(x=0.01,0.02)$ is shown in Figure 4. A clear hysteresis loop is observed for 2 at.\% $\mathrm{Fe}$ doped $\mathrm{NiO}$ as shown. The appearance of hysteresis loop at room temperature could be due to the ferromagnetic interaction amongst the $\mathrm{Fe}$ ions

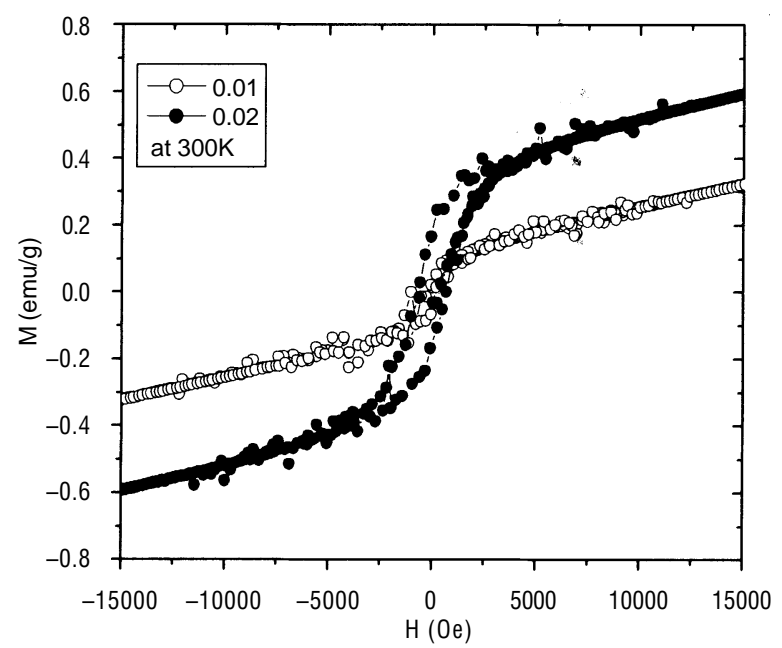

Figure 4. Field dependence magnetization curve for $x=0.01$ and 0.02 at room temperature.

mediated by the holes, which are created due to interstitial substitution of $\mathrm{Fe}$, which leads to 5 holes for each $\mathrm{Fe}^{3+}$ substitution. The hystersis loop does not saturate up to $40 \mathrm{KOe}$. It is a common feature that the magnetization of fine particles does not saturate. Kodama et al [6] have observed the unsatuartion of magnetization up to a field of $60 \mathrm{KOe}$ for $31.5 \mathrm{~nm} \mathrm{NiO}$ particle at $5 \mathrm{~K}$. In our case the particle as depicted from Williamson-Hall plot is larger than the critical limit to see finite size effect. Rather, 
the magnetic inhomogeneity, as suggested from the anomaly of FC magnetization at $315 \mathrm{~K}$ may contribute to the observed unsaturation in the hystersis plot.

\section{Conclusion}

In $\mathrm{Fe}$ doped $\mathrm{NiO}$ powder prepared by coprecipitation from nitrate precursors, we show that the size of crystallites motonically decrease with increasing $\mathrm{Fe}$ concentration. The strain on the other hand increases with increasing $\mathrm{Fe}$ and attains a maximum value for 3 at.\% $\mathrm{Fe}$ in $\mathrm{NiO}$. Beyond 3 at.\% the strain decreases indicating strain relaxation due to phase segregation and formation of $\gamma-\mathrm{Fe}_{2} \mathrm{O}_{3}$. The particles are larger than the threshold value below which finite size effect dominates. The observed magnetic behaviour therefore is a consequence of $\mathrm{Fe}$ induced magnetic ordering in the bulk of $\mathrm{NiO}$. In addition some amount of magnetic inhomogeneity also contributes to the magnetic characteristics of $\mathrm{Fe}$ doped $\mathrm{NiO}$ as evidenced from the temperature dependent magnetization study under field cooled and zero field cooled conditions.

\section{Acknowledgment}

We acknowledge the UGC-DAE CSR, Indore for providing magnetic measurement facility.

\section{References}

[1] H Ohno Science 281951 (1998); S A Wolf, D D Awschalom, R A Buhrman, J M Daughton, S Von Molnar, M L Roukes, A Y Chtchelkanova and D M Treger Science 2941488 (2001)

[2] T Dietl, H Ohno, F Matsukura, J Cibert and D Ferrand Science 2871019 (2000)

[3] J Wu, C-W Nan, Y Lin and Y Deng Phys. Rev. Lett. 89217601 (2002)

[4] O Bidault, M Maglione, M Actis and M Kchikech Phys. Rev. B52 4191 (1995)

[5] J Wang, J Cai, Y-H Lin and C-W Nan Appl. Phys. Lett. 87202501 (2005)

[6] R H Kodama, S A Makhlouf and A E Berkowitz Phys. Rev. Lett. 791393 (1997)

[7] J Nowotny and M Rekas Solid State lonics 12253 (1984)

[8] P D Battle, A K Cheetham and G A Gehring J. Appl. Phys. 507578 (1979)

[9] M Kurzawa and A Blonska-Tabero J. Therm. Anal. Calorim. 7765 (2004)

[10] W Schweika, A Hoser, M Martin and A E Carlsson Phys. Rev. B51 15771 (1995)

[11] A B Anderson, R W Grimes and A H Heuer J. Solid State Chem. 55353 (1984)

[12] F HaaB, T Buhrmester and M Martin Phys. Chem. Chem. Phys. 34806 (2001)

[13] G K Williamson and W H Hall Acta Metallur. 122 (1953)

[14] T Bitoh, K Ohba, M Takamatsu, T Shirane and S Chikazawa J. Phys. Soc. Jpn. 641305 (1995)

[15] W Luo, S R Nagel, T F Rosenbaum and R E Rosensweig Phys. Rev. Lett. 672721 (1991)

[16] B Martinez, X Obradors, L I Balcells, A Rouanet and C Monty Phys. Rev. Lett. 80181 (1998)

[17] S Dhar, O Brandt, A Trampert, K J Friedland, Y J Sun and K H Ploog Phys. Rev. B67 165205 (2003)

[18] S A Makhlouf, F T Parker, F E Spada and A E Berkowitz J. Appl. Phys. 815561 (1997)

[19] J Jeong, S Lee, J Kim and S Shin Phys. Stat. Sol.(b) 2411593 (2004) 